\title{
Evolution of low-mass star and brown dwarf eclipsing binaries
}

\author{
G. Chabrier, J. Gallardo, and I. Baraffe
}

\author{
École Normale Supérieure de Lyon, CRAL (UMR CNRS 5574), Université de Lyon, France \\ e-mail: chabrier@ens-lyon.fr
}

Received 23 April 2007 / Accepted 26 June 2007

\begin{abstract}
Context. We examine the evolution of low-mass star and brown dwarf eclipsing binaries. These objects are rapid rotators and are believed to shelter large magnetic fields.

Aims. We suggest that reduced convective efficiency, due to fast rotation and large field strengths, and/or to magnetic spot coverage of the radiating surface significantly affect their evolution, leading to a reduced heat flux and thus larger radii and cooler effective temperatures than for regular objects.

Methods. We have considered such processes in our evolutionary calculations, using a phenomenological approach.

Results. This yields mass-radius and effective temperature-radius relationships in agreement with the observations. We also reproduce the effective temperature ratio and the radii of the two components of the recently discovered puzzling eclipsing brown dwarf system.

Conclusions. These calculations show that fast rotation and/or magnetic activity may significantly affect the evolution of eclipsing binaries and that the mechanical and thermal properties of these objects depart from the ones of non-active low-mass objects. We find that, for internal field strengths compatible with the observed surface value of a few kiloGauss, convection can be severely inhibited. The onset of a central radiative zone for rapidly rotating active low-mass stars might thus occur below the usual $\sim 0.35 M_{\odot}$ limit.
\end{abstract}

Key words. convection - stars: activity - stars: binaries: eclipsing - stars: evolution - stars: low-mass, brown dwarfs - stars: rotation

\section{Introduction}

Low-mass stars (LMS), i.e., M-type stars in the present context, represent an overwhelming fraction of the Galactic stellar population (Chabrier 2003). Observational determination of their mass-radius relationship provides a stringent testing of the theoretical description of their structure and evolution. Although observations of this relationship agree well with the theoretical predictions in a large number of cases (Ségransan et al. 2003; Chabrier et al. 2005), the radius determinations of eclipsing binaries (EBs) depart significantly $(\sim 10-15 \%)$ from these predictions (Torres \& Ribas 2002; Chabrier et al. 2005). These discrepancies are unlikely to be due to inaccurate equation of state or opacities, whose treatment is relatively well mastered for $m \gtrsim 0.4 M_{\odot}$ and $T_{\text {eff }} \gtrsim 4000 \mathrm{~K}$ (Chabrier \& Baraffe 2000, CB00). Furthermore, active LMS are observed to be redder, i.e. cooler, than the other LMS (Hawley et al. 1996). These particular behaviours pose a challenge to theorists. On the other hand, the majority of LMS, notably EBs, are fast rotators and exhibit strong persistent coronal (X-ray) and chromospheric $\left(\mathrm{H}_{\alpha}\right)$ activity or large flares (Gizis et al. 2002), indicating the presence of a strong magnetic field (Donati et al. 2006). In this Letter, we suggest that the discrepancy between the observed mass-radius relationship of EBs and of other LMS does not stem from inaccurate equation-of-state or opacity problems, but is due to a rotation or magnetic field induced reduction of the efficiency of large-scale thermal convection in their interior, leading to less efficient heat transport. We show that the reduction of the star's radiating surface due to magnetic spot coverage also yields a smaller $T_{\text {eff }}$ and a larger radius.

\section{Effect of rotation and magnetic field}

The EBs and, more generally, active LMS are fast rotators with rotation periods of $P \lesssim 3$ days and rotation velocities of $v_{\text {rot }} \gtrsim 10 \mathrm{~km} \mathrm{~s}^{-1}$ (Delfosse et al. 1998; Reid et al. 2002; Mohanty \& Basri 2003). Typical convective time scales in their interior are $t_{\text {conv }} \sim \frac{H_{\mathrm{P}}}{v_{\text {conv }}} \sim \frac{10^{9}}{\left(10^{2}-10^{3}\right)} \sim 10^{6}-10^{7} \mathrm{~s}$, where $H_{\mathrm{P}} \sim R_{\star}$ is the pressure scale height (CB00). This yields Rossby numbers $R o=\frac{P}{t_{\text {conv }}} \lesssim 10^{-2}$. At a very small Rossby number, the Proudman-Taylor theorem enforces fluid motions to columnar or sheet-like structures with a characteristic length scale perpendicular to the rotation axis that is much smaller than the one along the rotation axis (of the order of $R_{\star}$ ). Although the conditions of this theorem do not exactly apply for spherical objects with finite viscosity, rotation-dominated convective motions are severely affected by rotation, leading to highly anisotropic patterns with large-scale fluid motions being confined to columns along the rotation axis and motions in the other directions being strongly inhibited (Zhang \& Jones 1997). As a whole, this reduces the characteristic length scale along these directions and the mean convective velocity amplitudes, thus the efficiency of thermal convection to transport the internal heat flux.

On the other hand, fully ionised interiors of LMS are excellent electrical conductors. The interaction between the magnetic field and the fluid motions induces an electrical current and a Lorentz force, which in turn affects the motions. In the presence of a magnetic field, the anisotropy of rotationdominated convection is reduced and the large-scale flows reach the so-called MAC (Magnetic, Archimedean, Coriolis) 
balance between the buoyancy, Coriolis, and Lorentz forces (Starchenko \& Jones 2002). Equipartition between the buoyancy force, $\sim \rho g \delta(\Delta T / T)$ (where $\delta=-\left(\frac{\partial \ln \rho}{\partial \ln T}\right)_{P}$ and $\Delta T$ is the temperature excess over a pressure scale height), and the Coriolis force, $\sim 2 \rho \Omega v_{\text {conv }}$ (where $\Omega=\frac{v_{\text {rot }}}{R_{\star}}$ is the star angular velocity), yields

$v_{\mathrm{conv}} \approx \frac{g \delta \Delta T}{T \Omega}$

for the typical average large-scale velocity. For $\Omega \sim 10^{-4} \mathrm{~s}^{-1}$, typical values of the various quantities over the LMS domain give $v_{\text {conv }} \sim 10-100 \mathrm{~cm} \mathrm{~s}^{-1}$, about a factor of 10 lower than the predictions of the usual mixing length theory.

There is presently no clear understanding of the interaction between convection and magnetic field under stellar interior conditions. We can, however, estimate the conditions for generation of a dynamo in LMS or BD interiors. The microscopic magnetic diffusivity of metallic hydrogen is $\eta \approx 10^{2}-10^{3} \mathrm{~cm}^{2} \mathrm{~s}^{-1}$. According to Ohm's law and Maxwell's equations (hydromagnetic induction equation), a magnetic field will decay unless a velocity field can counteract or balance the diffusive effects. For typical values of the convective velocity (see above), the characteristic magnetic Reynolds number over a star-size conducting region is $R_{\mathrm{m}}=v_{\text {conv }} R_{\star} / \eta \gg 100$. According to dynamo theory, $R_{\mathrm{m}}$ in LMS and BD interiors is thus large enough for dynamo to occur, providing both rotation and convection are present. Once the criterion for dynamo onset is satisfied, the field will grow and is supposed to equilibrate when the Lorentz and the Coriolis forces become comparable (Elsasser number of order unity), reaching the aforementioned MAC balance. This yields an amplitude for the internal field, $B_{\mathrm{eq}} \approx(8 \pi \bar{\rho} \eta \Omega)^{1 / 2} \gtrsim 10 \mathrm{G}$, for fast rotators $\left(v_{\text {rot }} \gtrsim 10 \mathrm{~km} \mathrm{~s}^{-1}\right)$.

At large $R_{\mathrm{m}}$, i.e. in the dissipationless regime, however, magnetic diffusion is mainly due to turbulent rather than molecular diffusion, with $\eta \equiv \eta_{t} \sim l v_{\text {conv }}{ }^{1}$. In that case, non-linear saturation occurs when turbulence, enhancing the diffusive processes, is strong enough to reduce $R_{\mathrm{m}}$ down to the critical value for dynamo action, $R_{\mathrm{m}} \gtrsim 50$. This yields typical magnetic length scales $l \lesssim R_{\star} / 50$, and thus $B_{\mathrm{eq}} \approx$ a few $10^{4} \mathrm{G}$ for LMS average conditions, in good agreement with surface field determinations of a few kG (Donati et al. 2006; Reiners \& Basri 2007). This corresponds to Alfven velocities $v_{A}=\frac{B}{\sqrt{4 \pi \rho}} \gtrsim v_{\text {conv }}$, so that for such strong fields the Lorentz force will impede the convection by reducing the flow speed. The main effect of a strong magnetic field is to inhibit motions across it in comparison with motions along it. An ideally conducting fluid is tied to the fluid lines. In a fluid of finite conductivity, motion across the field is possible at a rate governed by the conductivity. In a highly conductive medium with a strong magnetic field, the motion will be slow. Stevenson's (1979) stability analysis shows that the combination of fast rotation $(R o \lesssim 0.1)$ and a magnetic field with finite diffusivity enhances convection, because of the reduction by the Lorentz force of the flow anisotropy due to the Proudman-Taylor constraint. Stevenson's approach, however, applies to planar geometry, i.e. thin convection zones, and to uniform density and magnetic fields and is likely to break down for large (star-size) convective zones and strong fields. Magneto-convection 3D simulations indeed show that the magnetic field inhibits the magnitude of the velocity fluctuations and reduces the heat flux (Stein et al. 1992). On the basis of

1 Observations of sunspot decay indeed suggest that the solar surface diffusivity is many orders of magnitude higher than the atomic value. the aforementioned field strength values, it seems unavoidable to suppose that, even though the magnetic field will not necessarily stabilise the fluid against convection in a fluid of finite electrical resistivity, it will cause a serious reduction of convective efficiency.

These estimates, on the other hand, show that the magnetic pressure in LMS or BD interiors, $\frac{B^{2}}{8 \pi} \lesssim 10^{7} \mathrm{dyn}^{-2}$, is orders of magnitude lower than the gas pressure, $P_{\mathrm{g}} \gtrsim$ $10^{12}-10^{16} \mathrm{dyn}_{\mathrm{cm}^{-2}}$ (CB00), and it can be safely ignored in the internal structure equations. Indeed, if the fluid is convectively unstable, the ratio of the magnetic pressure inside the flux tube over the surrounding mean gas pressure is expected to be of the order of the superadiabaticity, $\frac{\left(B^{2} / 8 \pi\right)}{P_{\mathrm{g}}} \lessgtr\left(\nabla-\nabla_{\mathrm{ad}}\right) \lessgtr 10^{-7}$ (Gough \& Tayler 1966; Meyer 1994). An estimate for complete inhibition of convection is obtained when the Lorentz force is strong enough to balance the buoyancy force:

$$
\frac{B^{2}}{4 \pi l} \gtrsim \rho g \delta\left(\nabla-\nabla_{\mathrm{ad}}\right),
$$

where $l \ll R_{\star}$ is the aforementioned characteristic magnetic length scale in a dynamo turbulent medium. This yields field amplitudes of the order of $10^{4} \mathrm{G}$, comparable to the value of $B_{\text {eq. }}$. Magnetic fields can thus in principle severely inhibit convection in the interior of active LMS and BDs. The criterion (2) for stability against convection in the presence of a magnetic field is similar to the one derived by Stevenson in the dissipationless regime and by Gough \& Tayler (their Eq. (1.2)), except for the reducing factor $\sim l / R_{\star}$ for the gas pressure term (assuming $\nabla P_{\mathrm{g}} \sim P_{\mathrm{g}} / R_{\star}$ ). The Gough and Tayler approach, however, is primarily devoted to the study of surface spots, where the magnetic and gas pressures are comparable. Applying this criterion to LMS interiors yields field strengths $\gtrsim 10^{7} \mathrm{G}$ for the inhibition of convection in the core of a $0.3 M_{\odot}$ star (Mullan \& MacDonald 2001). It seems rather difficult to generate such strong fields. Together, near-equipartition (within a factor $\sim 10$ ) between turbulent and magnetic energy, $B^{2} / 8 \pi \sim$ $\rho v_{\text {conv }}^{2}$, and the fact that $L(r) \propto 4 \pi r^{2}\left(\rho v_{\text {conv }}^{3}\right)$ is a slowly varying quantity, yield an amplification factor $\sim 10-100$ from the surface to the central regions. A full field of several megaGauss would thus be in super-equipartition and, if confined to the interior, would be unstable (Elsasser numbers $\gg 1$ ) (Tayler 1973; Markey \& Tayler 1973). Therefore, it does not seem realistic to apply the Gough-Tayler criterion to the entire stellar structure, in particular for uniformly dense objects like LMS and BDs.

A real picture of a magnetised, convectively unstable medium is cooling flows along the magnetic flux tubes passing through the convective medium. Part of the thermal flux, confined to the tubes, is thus carried by diffusion. This contraint on the flow patterns leads to a substantial reduction in the transport of energy and reduces the maximum possible heat flux. Given the absence of a proper treatment of heat transport in a magnetised medium for LMS conditions, which requires multidimensional simulations over a characteristic convective length scale, and given the exploratory nature of this Letter, we decided to stick to a minimalist approach, focusing on the reduced efficiency of global thermal convection due to the presence of strong rotation and/or magnetic fields in EBs. Such an approach is based on the phenomenological representation of this network of diffusive tubes surrounded by impeded field-free convective regions as a global convective system - since the large Reynolds numbers illustrate the overwhelming importance of macroscopic motions over microscopic diffusive processes - with reduced 


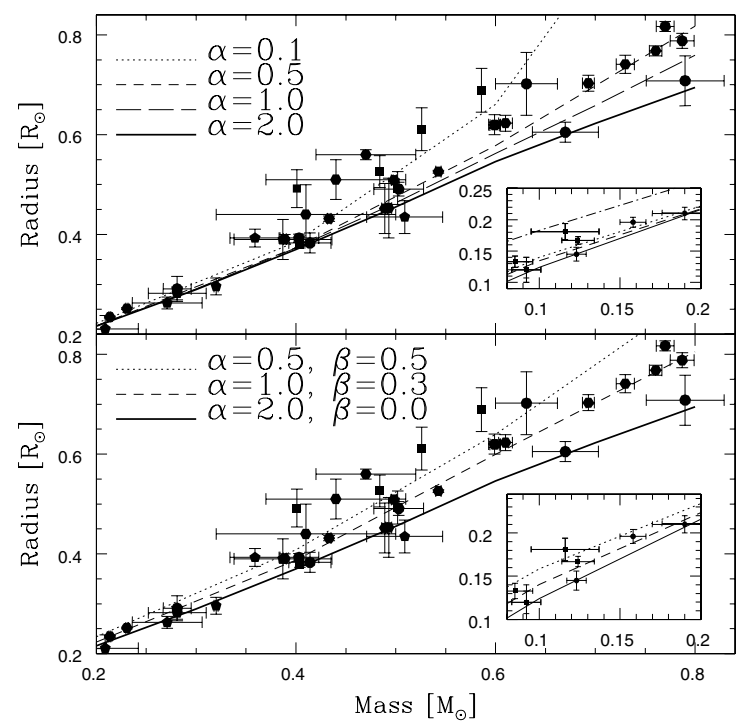

Fig. 1. Mass-radius relationships for $1 \mathrm{Gyr}$ old LMS for various values of the mixing length parameter $\alpha=l / H_{\mathrm{P}}$ (a) upper panel) and of the fractional surface area $\beta$ covered with cool spots (b) lower panel). Inset upper panel: dash-dot line: $\alpha=2,10^{8} \mathrm{yr}$. Solid thick line: standard case, no spot coverage. Low-mass eclipsing binary observational determinations are displayed with their $1 \sigma$ error bars. The observational data are from Torres et al. (2006), Berger et al. (2006), López-Morales (2007), and Beatty et al. (2007).

efficiency. In the framework of the standard MLT formalism, this translates into a mixing length parameter $\alpha=l / H_{\mathrm{P}}<1$.

\section{Effect of spot coverage}

The strong chromospheric and coronal emission in LMS of spectral types $\gtrsim \mathrm{M} 2-\mathrm{M} 3$, with $L_{\mathrm{H}_{\alpha}}$ and $L_{\mathrm{X}}$ about 100 times the solar value (Gizis et al. 2002; Mohanty \& Basri 2003), can be associated with an average large fraction of the radiating surface being covered with magnetic spots. Cool spots are the illustration of the inhibition of energy transport by convective motions (buoyancy) in a (rotating) highly conducting medium, but they also illustrate the fact that the field is not strong enough to suppress convection completely over the entire structure. This brings some justification to our admitedly simplistic approach where the aim is to explore the effect of reduced convective efficiency in EBs. We denote $\beta=S_{\mathrm{s}} / S_{\star}$ as the (time-averaged) fraction of the stellar surface covered by cool spots, $\mathcal{F}_{\mathrm{s}}$ the total flux emerging from the spots, and $\mathcal{F}_{\star}=\sigma T_{\text {eff }}{ }_{\star}$ the one associated with a spot-free star of effective temperature $T_{\text {eff }}$. Cool spots imply $\mathcal{F}_{\mathrm{s}}<\beta \mathcal{F}_{\star}$. The total flux is

$\mathcal{F}=(1-\beta) \mathcal{F}_{\star}+\mathcal{F}_{\mathrm{s}}<\mathcal{F}_{\star}$.

Cool spot coverage thus yields an effective temperature $T_{\text {eff }}<$ $T_{\text {eff } \star}$, a consequence of the reduced heat flow reaching the surface (Cowling 1976; Stein et al. 1992). Given the presently undetermined spot temperature for LMS and the exploratory nature of the present calculations, we simply assumed black (zero-temperature) spots. The effect of spot coverage for a given value of $\beta$ is thus an upper limit.

\section{Results}

\subsection{Effect of reduced convective efficiency}

Figure 1a portrays the $m-R$ relationship for LMS at $1 \mathrm{Gyr}^{2}$ for various values of the mixing length parameter $\alpha$. Observed radii

\footnotetext{
${ }^{2}$ Objects with $m \geq 0.09 M_{\odot}$ are on the ZAMS at this age (CB00).
}

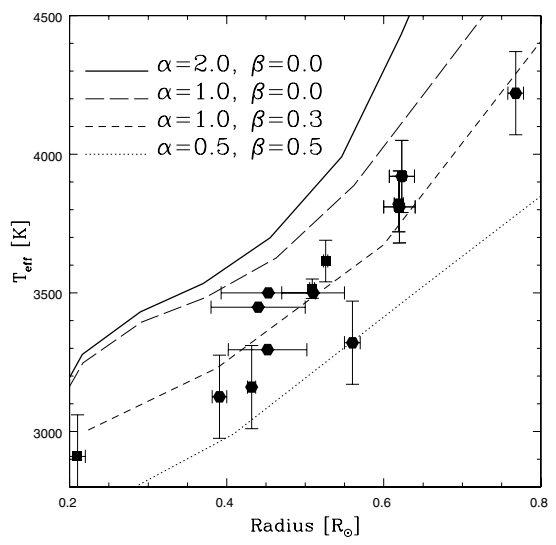

Fig. 2. Effective temperature vs. radius at $1 \mathrm{Gyr}$ for various values of $\alpha$ and $\beta$. Solid thick line: standard case, no spot coverage.

of EBs are displayed as well. Objects below $\sim 0.35 M_{\odot}$ are fully convective (Chabrier \& Baraffe 1997); convection is nearly adiabatic, so changing $\alpha$ for these objects has a modest impact. Since superadiabaticity increases with mass, in particular in the outermost regions of the star, reducing the convective efficiency, i.e. decreasing $\alpha$, for higher masses leads to an increasingly greater effect. The reduced convective flux requires a larger fraction of the heat to be transported by radiation in the outermost regions, yielding a steeper outer thermal gradient and thus a cooler $T_{\text {eff }}$. The immediate consequence is to decrease the luminosity and thus the central temperatures, i.e. the nuclear energy production needed to maintain thermal equilibrium. This in turn yields an expansion of the star, i.e. a larger radius. For BDs, the reduced heat flux implies a slower contraction rate.

We have also explored the fate of a $0.3 M_{\odot}$ star when drastically reducing the convective flux in the interior $(\alpha=0.05)$ as a result of condition (2). The star indeed develops a stable inner radiative zone $\left(\nabla_{\text {rad }}<\nabla_{\text {ad }}\right)$ over $\sim 20 \%$ of its mass, yielding a $\sim 7 \%$ larger radius and a cooler $T_{\text {eff }}$.

\subsection{Effect of spot coverage}

Figure $1 \mathrm{~b}$ illustrates the effect of surface spot coverage. As mentioned above, spot coverage yields a smaller heat flux output, i.e. cooler $T_{\text {eff }}$ and thus larger radii (since $L$ is unaffected) at a given age compared with spot-free objects. A spot coverage fraction $\beta \sim 30-50 \%$, for a value of $\alpha=1$, can by itself reproduce most of the observed EB radii within $1 \sigma$.

Figure 2 displays the effective temperature as a function of the radius for low-mass EBs for different values of $\alpha$ and $\beta$. Both reduced convective efficiency and spot coverage yield larger radii and cooler $T_{\text {eff }}$ compared with regular objects and provide a simple explanation for the particular mechanical and thermal properties of EBs. Figure 3 portrays $m-R$ relations for the recently discovered eclipsing BD system (Stassun et al. 2006). A solution with $\alpha=0.5, \beta=0.5$ for the most massive object $\left(m_{1}=0.054 M_{\odot}\right)$, and $\alpha=1, \beta=0.2$ for the least massive one $\left(m_{2}=0.034 M_{\odot}\right)$ yields radius values within the error bars and a temperature ratio $T_{\mathrm{eff} 2} / T_{\mathrm{eff} 1}=2440 \mathrm{~K} / 2320 \mathrm{~K}=1.05$, in agreement with the observational determination, for the age of the system $\sim 10^{6} \mathrm{yr}^{3}$. This possible solution suggests that the evolution

\footnotetext{
3 The larger $T_{\text {eff }}$ determinations obtained by Stassun et al. (2006) with the usual $\mathrm{Sp}-T_{\text {eff }}$-colour and $\mathrm{Sp}$-gravity relations suggest that these relations cannot be applied to active objects and to stellar surfaces exhibiting magnetic spots.
} 


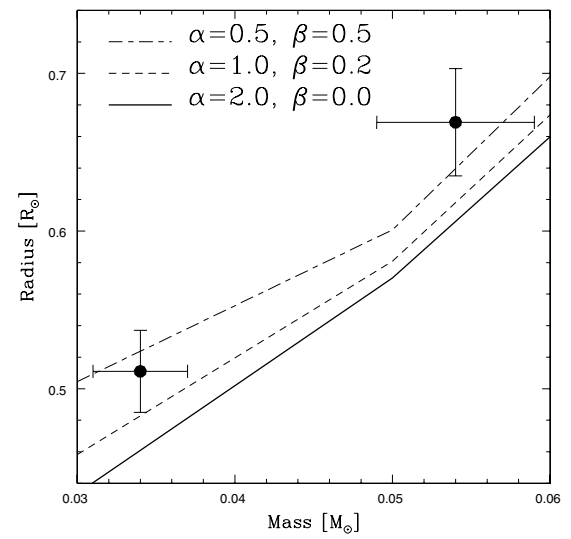

Fig. 3. Same as Fig. 1 for the eclipsing BD system (Stassun et al. 2006) for different values of $\alpha$ and $\beta$.

of the most massive BD has been significantly affected by the presence of a strong magnetic field, whereas the least massive BD has been less affected. Indeed, BDs with spectral type $\gtrsim \mathrm{M} 8-\mathrm{M} 9$, i.e. $T_{\text {eff }} \lesssim 2400 \mathrm{~K}$, are rapid rotators and are believed to shelter large-scale magnetic fields in spite of showing no sign of persistent activity (Mohanty \& Basri 2003; Chabrier \& Küker 2006). The reason invoked for this is the low electrical conductivity and thus the lack of substantial current generation in their atmosphere (Mohanty et al. 2002; Reiners \& Basri 2007). Spot coverage for cool ( $\left.T_{\text {eff }} \lessgtr 2400 \mathrm{~K}\right)$ BDs is thus expected to be small, even in the presence of a strong magnetic field. A possible, although speculative, explanation for the BD system of interest is that the most massive object was originally hot enough (its $T_{\text {eff }}$ for $\alpha=2, \beta=0$ is $T_{\text {eff }}=2800 \mathrm{~K}$ ) for significant spot coverage to occur, whereas the least massive BD remained too cool for atmospheric coupling between the magnetic field and the gas to be significant, yielding less spot coverage.

\section{Conclusion}

In this paper, we have examined the consequences of (i) inhibiting convection due to rotation and/or internal magnetic field and (ii) the presence of surface magnetic spot coverage, on the evolution of LMS and BDs. We have focused on the particular case of EBs. Our approach is phenomenological and the present paper makes no claim to present a consistent description of the effect of a large magnetic field on the evolution of dominantly convective objects. A proper approach to such a complex process for LMS interior conditions requires presently unavailable numerical tools. Our calculations show that rotation or magnetic field induced inhibition of convection leads to a reduced heat flow and thus significantly larger radii and cooler $T_{\text {eff }}$ than for regular objects. Spot coverage of the stellar radiating surface by itself has a major impact on the evolution. Either one or a combination of these effects explains the observed mechanical and thermal properties of EBs. Work is under way to explore the spot modulation in EBs and to derive observational values of the spot coverage fraction $\beta$ for M-stars (Morales et al., in preparation). A spot coverage fraction $\beta \sim 30-50 \%$, however, is consistent with observations of rapidly rotating, more massive active stars (Jeffers et al. 2005). These calculations strongly suggest that the evolution of EBs, or of very magnetically active LMS and BDs, differs noticeably from the one of objects for which rotational or magnetic effects are negligible, a fact supported by observational analysis (Morales et al. 2007). This should be taken into consideration when comparing observations with theoretical models. Global averaging of the mass-radius relationship for LMS is thus incorrect and highly misleading. We show that the puzzling eclipsing brown dwarf system that was recently discovered, with the more massive companion being the cooler one, can be explained if the evolution of the most massive object has been strongly affected by magnetic effects.

We suggest that internal field strengths consistent with the observed surface value, about a few $\mathrm{kG}$, might be sufficient for severely inhibiting convection in parts of LMS interiors, pushing the limit for the onset of an inner radiative zone below $\sim 0.35 M_{\odot}$, with important consequences for the field geometry (Chabrier \& Küker 2006; Donati et al. 2006).

\section{References}

Beatty, T. G., Fernández, J. M., Latham, D. W., et al. 2007, ApJ, 663, 573 Berger, D. H., Gies, D. R., McAlister, H. A., et al. 2006, ApJ, 644, 475 Chabrier, G. 2003, PASP, 2003, 115, 763

Chabrier, G., \& Baraffe, I. 1997, A\&A, 327, 1039

Chabrier, G., \& Baraffe, I. 2000, ARA\&A, 38, 337 (CB00)

Chabrier, G., \& Küker, M. 2006, A\&A, 446, 1027

Chabrier, G., Baraffe, I., Allard, F., \& Hauschildt, P. 2005, in Resolved Stellar Populations, ed. D. Valls-Gabaud [arXiv: astro-ph/0509798]

Cowling, T. G. 1976, MNRAS, 177, 409

Delfosse, X., Forveille, T., Perrier, C., \& Mayor, M. 1998, A\&A, 331, 581

Donati, J.-F., Forveille, T., Cameron, A., et al. 2006, Science, 311, 633

Gizis, J., Reid, I., \& Hawley, S. 2002, AJ, 123, 3356

Gough, D., \& Tayler, R. 1966, MNRAS, 133, 85

Hawley, S. L., Gizis, J. E., \& Reid, N. I. 1996, AJ, 112, 2799

Jeffers, S. V., Cameron, A., Barnes, J. R., Aufdenberg, J., \& Hussain, G. 2005, ApJ, 621, 425

López-Morales, M., 2007, ApJ, 660, 732

Markey, P., \& Tayler, R. 1973, MNRAS, 163, 77

Meyer, F. 1994, in Cosmical Magnetism, ed. D. Lynden-Bell (Kluwer)

Mohanty, S., \& Basri, G. 2003, ApJ, 583, 451

Mohanty, S., Basri, G., Shu, F., Allard, F., \& Chabrier, G. 2002 ApJ, 571, 469

Morales, J. C., Ribas, I., \& Jordi, C. 2007, A\&A, submitted

Mullan, D., \& MacDonald, J. 2001, ApJ, 559, 353

Reid, N. I., Gizis, J. E., \& Hawley, S. L. 2002, AJ, 124, 2721

Reiners, \& Basri, G. 2007, ApJ, 656, 1121

Ségransan, D., Kervella, P., Forveille, T., \& Queloz, D. 2003, A\&A, 397, L5

Starchenko, S. V., \& Jones, C. A. 2002, Icarus, 157, 426

Stassun, K. G., Mathieu, R. D., \& Valenti, J. A. 2006, Nature, 440, 311

Stein, R., Brandenburg, A., \& Nordlund, A. 1992, ASP. Conf. Ser., 26, 148

Stevenson, D. J. 1979, Geophys. Astrophys. Fluid Dyn., 12, 139

Tayler, R. 1973, MNRAS, 161, 365

Torres, G., \& Ribas, I. 2002, ApJ, 567, 1140

Torres, G., Lacy, C., Marschall, L., Sheets, H., \& Mader, J., 2006, ApJ, 640, 1018

Zhang, K., \& Jones, C. A. 1997, Geophys. Res. Lett., 24, 2869 